\title{
IDO inhibitors could change tack to treat metabolic disorders
}

\section{Inhibitors of indoleamine}

2,3-dioxygenase (IDO) have been investigated in the clinic for their potential to increase the effectiveness of immunotherapies in cancer. Following a recent late-stage clinical failure, many companies have binned their IDO programmes. An article from Soraya Taleb's lab now demonstrates that IDO inhibitors could be useful in regulating metabolic disorders, as these molecules alter the metabolism of the gut microbiota in mice.
The authors began by investigating the role of IDO in obesity. "The activity of this enzyme is increased in the intestinal IDO may alter the metabolites available to the gut microbiota
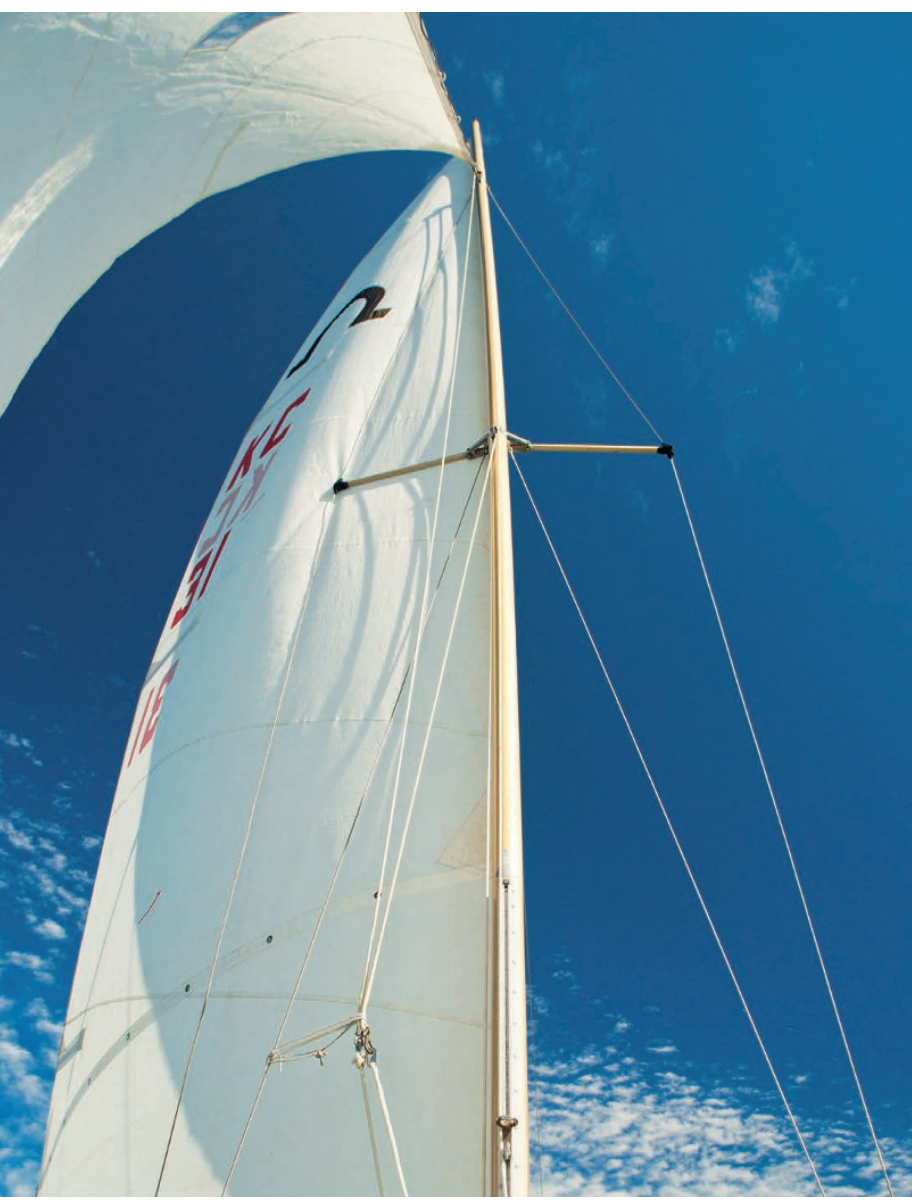

Alexander McClearn/Alamy Stock Photo context of cardiovascular diseases and obesity, and this increase in patients with cardiovascular diseases is predictive of worse cardiovascular outcome," explains Taleb. They found that Ido $1^{-/-}$mice that were fed a high-fat diet (HFD) gained less weight and had a lower fat mass, better glucose and insulin tolerance and less macrophage infiltration into fat tissue than wildtype (WT) mice did. Further analysis attributed the difference in fat mass to a higher metabolic rate in muscles. Treatment with L-1-methyl-tryptophan (1MT), an IDO inhibitor, concurrent with a HFD had a similar effect on insulin and glucose tolerance to that in the knockout.

Previous work had suggested that IDO inhibitors could alter obesity through effects on T cells, as IDO generates kynurenine metabolites that induce regulatory $\mathrm{T}$ cells, which have been shown to protect against obesity. Surprisingly, chimeric mice that lacked IDO in the bone marrow compartment had no alterations in metabolic measurements relative to WT mice, whereas Ido1 $1^{-/}$mice reconstituted with bone marrow from WT mice recapitulated the phenotype of the $I d o 1^{-/}$mice. As IDO is expressed in the gastrointestinal tract, the authors hypothesized that intestinal IDO may alter the metabolites available to the gut microbiota.

Indeed, antibiotic treatment prevented both $\mathrm{Idol}^{-/-}$and WT mice from gaining weight on a HFD. $\mathrm{IdoI}^{-/-}$and WT mice that were co-housed had metabolic measurements similar to those of $I d o 1^{-/-}$mice housed by genotype, suggesting that the microbiota from $\mathrm{Idol}^{-/-}$mice is protective. Consistent with this hypothesis, oral gavage with the faeces of genetically obese mice $(o b / o b)$ that were fed 1MT improved the metabolic measurements of WT mice compared with oral gavage with faeces from untreated $o b / o b$ mice. Ido $1^{-/-}$and WT mice had different intestinal microbiota composition: HFD-fed WT mice had a higher Firmicutes:Bacteroidetes ratio than HFD-fed $I d o 1^{-/-}$mice did, a ratio that is associated with poor intestinal health.

Tryptophan can be metabolized either by IDO to produce kynurenine, or by gut bacteria to produce indole derivatives such as indole-3-acetic acid (IAA), a ligand for the aryl hydrocarbon receptor (AHR). Depletion of IDO through genetic or pharmacological methods increased the levels of IAA in the faeces. IAA-induced activation of AHR in intestinal immune cells increases the production of IL-17 and IL-22, and indeed the levels of these two cytokines were higher in HFD-fed Ido1 $1^{-/}$mice than in HFD-fed WT mice. Injection of an anti-IL-22 neutralizing antibody in HFD-fed $\mathrm{Idol}^{-/}$ mice abrogated the protective effects of IDO deficiency on obesity, insulin sensitivity and intestinal permeability.

Consistent with the observations in mice, people with obesity or type 2 diabetes had higher levels of kynurenine in their plasma and faeces and lower levels of IAA in their faeces.

These data suggest that altering the intestinal metabolism of tryptophan could have implications in the treatment of obesity. Taleb proposes repurposing IDO inhibitors in patients with cardiometabolic diseases, or supplementing obese patients with indole metabolites or with indole-producing bacteria. Megan Cully

ORIGINAL ARTICLE Laurans, L. et al. Genetic deficiency of indoleamine 2,3-dioxygenase promotes gut microbiota-mediated metabolic health. Nat. Med. https://doi.org/10.1038/s41591018-0060-4 (2018) 